\title{
LXIV. On certain functions resembling quaternions, and on a new imaginary in algebra
}

\author{
James Cockle Esq. M.A. \& T.S. Davies Esq. F.R.S.L. \& E.
}

To cite this article: James Cockle Esq. M.A. \& T.S. Davies Esq. F.R.S.L. \& E. (1848) LXIV. On certain functions resembling quaternions, and on a new imaginary in algebra, Philosophical Magazine Series 3, 33:224, 435-439, DOI: 10.1080/14786444808646139

To link to this article: http://dx.doi.org/10.1080/14786444808646139

Published online: 30 Apr 2009.

Submit your article to this journal $₫$

Џ Article views: 7

Q View related articles $\square$ 
LXIV. On certain Functions resembling Quaternions, and on a new Imaginary in Algebra. By James Cockie, Esq., M.A., Barrister-at-Law. In a Letter to T. S. Davies, Esq., F.R.S.L.\&E. \&C.*

To Richard Taylor, Esq., F.S.A. \&.c.

My dear Sir, Royal Military Academy, Woolwich, October 27, 1848.

T BELIEVE that Mr. Horner's attention was first directed 1 by me to the consideration of what he has happily termed "congeneric surd equations." This was done in consequence of a difficulty that $I$ had found in discussing such an equation which was accidentally brought before me; a difficulty which, from the friendship that had long subsisted between us, I very naturally referred to him. His reply was printed in the Philosophical Magazine about a dozen years ago; and it has received proper attention from those algebraists who look with due care to the fundamental principles of their science.

Almost every new difficulty in algebra is the precursor of more extended views respecting its principles; and often out of a case which may, at first sight, appear to be little more than a conundrum, very important improvements in our general theories often arise. All efforts to generalize our views, so as to include the "conundrum," deserve, therefore, attentive consideration; and for this reasun I send you for publication the accompanying letter, which $I$ have just received from your correspondent and my friend, Mr. James Cockle, of the Middle Temple.

I hope you will be able to give Mr. Cockle's letter an early place in your valuable Magazine, as it contains much that is suggestive of inquiry.

I remain,

Yours very truly, T. S. Davies.

\section{MY DEAR SIR,}

2 Church.Yard Court, Temple, October 26, 1848.

YOU are aware that the new algebraical symbol, which $\mathbf{I}$ 1 have recently discovered, was suggested to my mind by reflecting on the structure of congeneric surd equations-a subject on which $I$ have lately been much engaged. Did I require any excuse for throwing the present investigations into the form of a letter to you, $I$ should find it in the fact that the remarks of Horner on congenerics were put into a similar shape. (See Phil. Mag., S. 3, vol. viii. p. 43-50.)

* Communicated by Mr. Davies. 
In what follows, I propose to confine the term unreal to the imaginary quantities of ordinary algebra. When the word impossible is used, it must be understood as referring exclusively to expressions involving the new symbol. So that imaginary quantities constitute a genus which includes two species, viz. unreal quantities and impossible quantities.

Let

$$
1+i^{2}=0
$$

then $i$ is the simplest representation of unreal quantity; and, if

$$
I+\sqrt{j}=0,
$$

then $j$ is the simplest representation of impossible quantity. It is to be borne in mind that, in the latter equation, the radical is to be considered as essentially affected with the sign + .

Let $w, x, y, z$ be any real quantities, positive, negative, or zero; also let

$$
w+i x+j y+i j z=t
$$

then $t$ I call a tessarine, and $z, x, y, z$ its constituents. The latter term $I$ have adopted from the quaternion theory of Sir W. R. Hamilton.

This being premised, I sliall first proceed to prove, that, when a tessarine vanishes, its constituents are simultaneously zero.

Suppose then that $t=0$, and solve this last equation with respect to $j$; the result is

$$
j=-(w+i x) \div-(y+i z)
$$

that is to say, $j$ is capable of being linearly expressed in terms of $i$ and of real quantities, for we may readily reduce the above expression for $j$ to the form

$$
j=\alpha+i \beta \text {. }
$$

But, as may be proved from the principles of my theory of congeneric surd equations $*$, there is no linear relation between $i$ and $j$. Hence $\alpha$ and $\beta$ must each be of the form $0 \div 0$; and we see, in consequence, that when $t=0$, we have, necessarily,

$$
w=0, x=0, y=0 \text {, and } z=0 \text {. }
$$

The symbol $j$ is not capable of being identified with any of the imaginaries of the quaternion theory. It is a deduction from the principles of algebra rather than an invention. I shall not repeat my argument in favour of its admission as a new and independent symbol, because I think that I have put the question of the propriety of such admission beyond dispute at

* Mechanics' Magazine, vol. xlvii. pp. 151, 307, 331, 409; vol. xlviii. p. 181 ; vol. xlix. p. 364 . 
pp. 364-367 of the current (49th) volume of the Mechanics' Magazine; I shall rather go on with the development of the properties of expressions which involve it.

For convenience, I shall denote the product of $i$ and $j$ by $k$; I shall assume that $j^{2}$ is equal to unity. I say assume because, although I have reasons (not, however, free from objection, ) for making such a supposition, yet I wish to reserve to myself full liberty to modify the assumption, and to discuss $a b$ initio the symbol $j^{n}$ and its symbolic relation to $j$. Then the following system of relations will, together with the second equation given in this letter, furnish us with all the conditions requisite for the formation of a theory of these three imaginaries. That system is-

$$
\begin{aligned}
& i^{2}=-j^{2}=k^{2}=-1, \\
& i j=k, \quad j k=i, \quad k i=-j .
\end{aligned}
$$

Almost the first thing that strikes us, in examining the expression for a tessarine, is, that, under the above system, the product of two or more tessarines is a tessarine. Let us confine our attention to the case in which two only are multiplied together, and let the factors be $t$ and $t^{\prime}$, where

and

$$
t=w+i x+j y+k z
$$

$$
t^{\prime}=w w^{\prime}+i x^{\prime}+j y^{\prime}+k z^{\prime} \text {. }
$$

By actual multiplication, we obtain

Suppose that

$$
\left.\begin{array}{l}
t t^{\prime}=w w w^{\prime}+i^{2} x x^{\prime}+j^{2} y y^{\prime}+k^{2} z z^{\prime} \\
+i\left(w x^{\prime}+x w\right)+j k\left(y z^{\prime}+z y^{\prime}\right) \\
+j\left(w y^{\prime}+y w\right)+i k\left(x z^{\prime}+z x^{\prime}\right) \\
+k\left(w z^{\prime}+z w w^{\prime}\right)+i j\left(x y^{\prime}+y x^{\prime}\right) .
\end{array}\right\}
$$

and also that

$$
\left.\begin{array}{l}
w w w^{\prime}-x x^{\prime}+y y^{\prime}-z z^{\prime}=w w^{\prime \prime} \\
w x^{\prime}+x w^{\prime}+y z^{\prime}+z y^{\prime}=x^{\prime \prime}, \\
w y^{\prime}+y w^{\prime}-x z^{\prime}-z x^{\prime}=y^{\prime \prime}, \\
w z^{\prime}+z w^{\prime}+x y^{\prime}+y x^{\prime}=z^{\prime \prime},
\end{array}\right\}
$$

$$
z w^{\prime \prime}+i x^{\prime \prime}+j y^{\prime \prime}+k z^{\prime \prime}=t^{\prime \prime} \text {; }
$$

then we have, under the foregoing system of relations,

$$
t t^{\prime}=t^{\prime \prime}
$$

and, if we had three or more such factors as $t$, the result would still be a tessarine.

The next point that arises is, - what is the tessarine lare of moduli? or, more generally, what modular or quasi-modular 
relation or relations exist between the constituents of the factors and those of the product? Seeing that the operations of algebra necessarily and inevitably conduct us to tessarine formulæ, I cannot doubt but that some relations may be found, such as to enable us to extend the boundaries of analysis. I have not, however, time to pursue this inquiry further now - more particulariy as I wish to make one or two remarks upon other parts of the subject of this new imaginary $j$.

For instance, I would observe that the product of two or more factors of the form

$$
w+j y
$$

is of the same form; thus

where

$$
(w+j y)\left(w+j y^{\prime}\right)=w w^{\prime \prime}+j y^{\prime \prime},
$$

and

$$
r e^{\prime \prime}=\operatorname{rerco}+y y^{\prime}
$$

$$
y^{\prime \prime}=r y^{\prime}+y w^{\prime} \text {. }
$$

There are cases in which the impossible quantity $j$ altogether disappears from a result. Thus, if $\varepsilon$ be the base of the Napierian logarithms, we have

and

$$
\varepsilon^{j y}=1+j y+\frac{y^{2}}{2}+j \frac{y^{3}}{2.3}+\frac{y^{4}}{2.3 .4}+\& c .,
$$

hence

$$
\varepsilon^{-j y}=1-j y+\frac{y^{2}}{2}-j \frac{y^{3}}{2.3}+\frac{y^{4}}{2.3 .4}-8 \mathrm{c} . ;
$$

and

$$
\varepsilon^{j y}+\varepsilon^{-j y}=2\left(1+\frac{y^{2}}{2}+\frac{y^{4}}{2.3 .4}+8 \mathrm{c} .\right)
$$

$$
\frac{1}{2 j}\left(\varepsilon^{j y}-\varepsilon^{-j y}\right)=y+\frac{y^{3}}{2.3}+\& c . .
$$

This last is a very simple case; but the nature of such a Jetter as this obliges me to have recourse to illustration rather than extensive investigation.

I need not tell you how to read the second equation which occurs in this communication; still, I cannot help saying-to use words adopted on another occasion, by our friend Professor J. R. Young (Alg. 4th edit. p. 131),-that we must read that equation as meaning that unity together woith the plus [square-] root of $j$ is equal to zero, and NOT that unity together with a square-root of $j$ is equal to zero. The whole discussion is involved in the correct reading of that fundamental equation.

By way of conclusion, I will add that, if we had three inde- 
Prof. E. Wartmann's Seventh Memoir on Induction. 4.39

pendent imaginaries $i, j, k$, we should have to deal, not with tessarines, but with what might be called octrines. These lastmentioned expressions would be of the form

$$
w+i x+j y+k z+i j p+i k q+j k r+i j k s
$$

where $w, x, y, z, p, q, r, s$ are any real quantities. These quantities might be termed the constituents of the octrine, which could not vanish unless all its constituents were zero. So if we had four independent imaginaries (that is to say, imaginaries unconnected by any linear relation), the corresponding expression would consist of sixteen terms, and would have properties analogous to those of the tessarine and the octrine. I must here break off, but I remain, always,

My dear Sir,

$$
\text { Yours most truly, }
$$

James Cockle.

To T. S. Davies, Esq., F.R.S. L.\& E., \&c.\&c. \&c., Woolwich.

LXV. Seventh Memoir on Induction. ByM. Elie Wartmann, Professor of Natural Philosophy in the Academy of Geneva*.

[Continued from p. 278.]

$\$ \mathrm{XIX}$. On the relations of Electricity roith bodies in the spheroidal state, and on some properties of those bodies.

178. T $\mathrm{T}$ is a well-known fact that liquids are incapable of moistening solid surfaces sufficiently heated, or of mixing, in certain cases, with other liquids raised to a high temperature. The first accurate observation of this fact is attributed to Eller: it dates from 1746t. Ten years later, this phænomenon was studied by Leidenfrost, by whose name it is still designated $\ddagger$. Since then a number of experiments little known have been made in Germany $\$$; afterwards Italy $\mid$

- Communicated by the Author.

+ Histoire de l'Académie de Berlin, 1746, p. 42.

$\$$ De aque communis nonnullis qualitatibus: Duisburg, 1756.

$\$$ Among others we may cite Ziegler, Specimen de digestore Papini, 1765 . -Link, Beiträge zu' Physik und Chemie, No. 2, p. 11.--Kastner, Tromsdorff's Journal, vol. ix. p. 270. - Ersted, Gehlen's N. Allgem. Journ., vol. iii. p. 324.-Klaproth, Scherer's Allg. Journal de Chemie, vol. vii. p. 646 ; and Journal de Physique, 1802, p. 62.-Döbereiner, Schweig. Journ., vol. xxix. p. 43.-N. W. Fischer, Poggend. Annalen, vol. xix. p. 514, and vol. xxi. p. 163.-Buff, Pogg. Ann., vol. xxv. p. 591.-A. H. Emsmann, Pogg. Ann., vol. li. p. 444.-Frei, Kastner's Archiv, vol. iv. p. 57.-Erdmann, Journal für Praktische Chemie, vol. x. p. 334.-Böllger, Eine Sammlung eigener Erfahrungen, Versuche und Beobachtungen, No. 18, Francfort-sur-Mein, 1838.

|| Bellani, Giornale di Fisica di Brugnatelli, 1808, p. 779; Giornale di 